\title{
APLICACIÓN DE ANÁLISIS DISCRIMINANTE EN LA LIQUI- DEZ Y RENTABILIDAD DE LAS EMPRESAS CERTIFICADAS EN BASC EN PEREIRA-COLOMBIA'
}

\section{APPLICATION OF DISCRIMINANT ANALYSIS IN THE LIQUIDITY AND PROFITABILITY OF COMPANIES CERTIFIED IN BASC IN PEREIRA - COLOMBIA}

\author{
Tomás Fontalvo Herrera*, José Morelos Gómez**, Adel Mendoza Mendoza***
}

\begin{abstract}
Resumen
En este artículo de investigación se presentan los resultados obtenidos durante el desarrollo del proyecto evaluación del impacto de la certificación Business Aliance For Security Commerce (BASC) en la liquidez y rentabilidad de las empresas de Pereira. Se realiza inicialmente una descripción detallada de la evaluación de los sistemas organizacionales, la certificación BASC, los indicadores de liquidez, indicadores de rentabilidad, los criterios para la evaluación del impacto de la certificación BASC en la liquidez y rentabilidad de las empresas de Pereira y la utilización de la técnica análisis discriminante para diferenciar dos periodos establecidos 2008 y 2010, y su relación con la certificación BASC. Lo anterior, a través de una perspectiva propositiva y cuantitativa. De la función discriminante obtenida y de los estadísticos analizados se puede concluir que la certificación BASC en la función discriminante, incide positivamente en los indicadores financieros, Razón Corriente (RC), Margen Bruto ( MB) y Utilidad Operacional (UO).
\end{abstract}

Palabras clave: Certificación BASC, análisis discriminante, aplicación, procesos.

\section{Abstract}

This research paper presents the results obtained during the development of the research work: evaluation of the impact of the certification Business Aliance For Security Commerce (BASC) on liquidity and profitability of companies of Pereira; during this work, initially were performed: a detailed description of the evaluation of organizational systems, the BASC certification, liquidity indicators, indicators of profitability, the criteria for the evaluation of the impact of BASC certification on the liquidity and profitability of companies in Pereira and the use of discriminant analysis technique to distinguish two periods: 2008-2010, and its

Artículo recibido: 20/12/2012 Aprobado: 14/05/2013

1 Resultados de proyecto de investigación titulado "Evaluación del impacto de la certificación BASC en la liquidez y rentabilidad de las empresas de Pereira-Colombia. Investigación financiada por Vicerrectoría de Investigaciones, V convocatoria grupos de investigación categorizados en Colciencias año 2011. Grupo de Investigación Calidad y productividad Organizacional Integral. Universidad de Cartagena.

* PhD. Administración de Empresas. Jefe de departamento de Organización Industrial, Universidad de Cartagena. Email: tfontalvoh@ unicartagena.edu.co

** Magíster en Administración. Director de Programa de Administración Industrial, Universidad de Cartagena. Email: jmorelosg@unicartagena. edu.co

*** Magíster en Ingeniería Industrial. Profesor de Tiempo Completo, Universidad del Atlántico. Email: adelmendoza@mail.uniatlantico.edu.co 
relationship with the BASC certification. All this from a purposeful and quantitative perspective. From the discriminant function that was obtained, and from the statistic analyzed, it can be concluded that the BASC certification in the discriminant function, has a positive impact on financial indicators, Current Ratio (CR) Gross Margin (GM) and Operating Profit (UP).

Key words: BASC certification, discriminant analysis, application, processes.

\section{Introducción}

En este artículo se desarrolló una metodología para evaluar la aplicación de la certificación de la alianza de los negocios para la seguridad del comercio y su incidencia en la liquidez y rentabilidad, tomando para este estudio 60 empresas certificadas de la ciudad de Pereira, que presentaron sus estados financieros en la Superintendencia de Sociedades y la Cámara de Comercio de Pereira.

Con el fin de realizar una evaluación detallada de las empresas se utilizó el análisis discriminante tomando indicadores financieros y definiendo las variables dependientes e independientes que permitieron analizar la varianza dentro de los grupos y entre grupos; con lo que se evaluó el desempeño de las compañías certificadas en BASC de tal forma que se pudiera analizar la relación existente entre las empresas certificadas y la incidencia en los indicadores financieros seleccionados en el periodo 2008-2010.

Un estudio de esta naturaleza implica la revisión conceptual y teórica sobre los sistemas organizacionales, la certificación alianza de los negocios para la seguridad del comercio, los indicadores de liquidez, indicadores de rentabilidad, los criterios para evaluar el impacto de la certificación BASC, la técnica análisis discriminante y la distancia $D^{2}$ de Mahalanobis. A partir del marco teórico se elaboró un modelo que sirvió de fundamento para la evaluación de la aplicación de la alianza para la seguridad del comercio en la liquidez y rentabilidad de las empresas de Pereira.

\section{Marco de aplicación}

\subsection{Evaluación de sistemas organizacionales}

Criado y Calvo (2009), señalan que la Evaluación de organizaciones supone un cuerpo de conocimientos teóricos y metodológicos, así como un conjunto de habilidades aplicadas. Para esta Investigación este cuerpo de conocimientos los constituye los fundamentos de
Análisis Discriminante Multivariado (DMA), que permiten analizar la evaluación del impacto de la certificación BASC en la liquidez y rentabilidad de las empresas de Medellín certificadas en BASC. Asimismo, Martínez (2005) y Fernández (2002), indican que la evaluación significa estimar la magnitud de un hecho, proceso o servicio, en el cual el análisis debe enfocarse hacia el estudio cuidadoso que animan a los involucrados en la situación problemática, a la evaluación de las variables controlables (internas al sistema), a la evaluación de las variables incontrolables (ambiente), al estudio de las interrelaciones entre variables y la superación de los límites del sistema en la empresa, en el proceso de la evaluación del impacto de la certificación BASC.

\subsection{Certificación business aliance for security commerce}

WBO (2011), define el BASC (Business Anti-Smuggling Coalition o Coalición Empresarial Anticontrabando) como un programa de cooperación entre el sector privado y organismos nacionales y extranjeros, creado para fomentar un comercio internacional seguro.

Es un programa de carácter voluntario, otorga una certificación que confirma el trabajo de control en todos los procesos productivos, empaque, embarque y de transporte de la carga que va con destino al exterior, garantizando que la carga no tiene posibilidades de contaminación (contrabando y drogas) en ninguna etapa hasta llegar al destino final.

Estándar que con criterios específicos plantea requisitos que están asociados y contribuyen con la eficiencia y eficacia de las organizaciones donde se implementen. En esencia esta certificación se centra en mejorar los procesos internos de las organizaciones que los implementen. Ovalle y Ospina (2009), señala que todas las empresas que se dediquen al mercado exportador deben tener certificación BASC. La norma es avalada por el Gobierno colombiano, la aduana americana, la aduana francesa, entre otras. 


\subsubsection{Alcance de la certificación business aliance for security commerce}

Esta norma establece requisitos y da información sobre: El desarrollo de sistemas de Gestión en Control y Seguridad en el Comercio Internacional; los lazos con otras normas sobre sistemas de administración. Está diseñada para ser utilizada por organizaciones de todos los tamaños, independientemente de la naturaleza de sus actividades. Donde su aplicación sea proporcional a las circunstancias y necesidades de cada organización particular, añadiendo valor agregado a dichos elementos y generando rendimientos al sistema. Lo cual se evalúa en esta investigación a través del Análisis Discriminante Multivariado (DMA).

\subsection{Aplicación de la certificación business aliance for security commerce en la liquidez y rentabilidad de las empresas de Pereira.}

Al evaluar la aplicación de la certificación, en los ingresos y crecimiento de las empresas de Medellín, se puede adoptar y mejorar estándares o hacer ajustes en los sistemas organizacionales y específicamente en los implementados bajo la Norma BASC, de tal forma que reflejen eficiencia, eficacia, liquidez y rentabilidad de las organizaciones que los adopten.

\subsection{Análisis discriminante}

Es una técnica de Análisis Multivariante que procura encontrar relaciones lineales entre las variables continuas que mejor discriminen en los grupos categóricos previamente definidos. Mylonakis y Diacogiannis (2010) y Suárez (2000), señalan que el Análisis Discriminante es una técnica estadística, cuya finalidad es analizar si existen diferencias significativas entre grupos de objetos respecto a un conjunto de variables. Para el caso de esta investigación se analiza el comportamiento en dos periodos distintos, utilizando los indicadores financieros seleccionados para las empresas de Pereira que se encuentran certificadas.

\subsection{Distancia de Mahalanobis.}

Como criterio de selección de variables que mejor discriminan los indicadores de liquidez y rentabilidad en la certificación BASC en las empresas de Pereira, se utilizó la distancia $D^{2}$ de Mahalanobis. La regla de selección en este procedimiento es maximizar esta distancia multivariante entre los grupos a y $b$ la cual se define como:

$$
D=(n-k) \sum_{i=1}^{p} \sum_{j=1}^{p} W^{-1}{ }_{i j}\left(X_{i}^{(a)}-X_{i}^{(b)}\right)\left(X_{j}^{(a)}-X_{j}^{(b)}\right)
$$

Donde $n$ es el número de casos válidos, $k$ es el número de grupos, $X_{i}{ }^{(a)}$ es la media del grupo $b, y X_{i}{ }^{(b)}, y W_{i j}{ }^{-1}$ es un elemento de la inversa de la matriz de varianzascovarianzas intra-grupos. Siendo la variabilidad total de la forma

$$
T_{i j}=W_{i j}+V_{i j}
$$

La covarianza total es igual a la covarianza dentro de grupos más la covarianza entre grupos. Así, la probabilidad de que un objeto j, con una puntuación discriminante $D=\left(y_{\mathrm{j} 1}, \ldots, y_{\mathrm{jm}}\right)$ pertenezca al grupo i-ésimo se puede estimar mediante la regla de Bayes:

$$
P\left(K_{i} / D\right)=\frac{P\left(D / K_{i}\right) P\left(K_{i}\right)}{\sum_{i}^{m} P\left(D / K_{i}\right) P\left(K_{i}\right)}
$$

$P\left(K_{i}\right)$ es la probabilidad a priori y es una estima de la confianza de que un objeto pertenezca a un grupo si no se tiene información previa.

A continuación se estructura y propone el modelo de como la certificación para la alianza de los negocios y la seguridad comercial, afecta los indicadores de liquidez y rentabilidad en las empresas de Medellín, en los años 2008 y 2010.

\section{Metodología}

Para la realización de esta investigación se utilizaron los indicadores presentados a continuación, como se muestra en la Tabla 1.

\section{Población}

La población de esta Investigación está conformada por 12 empresas certificadas de alianza para la seguridad del comercio de la ciudad de Pereira, las cuales mantuvieron este estándar entre el año 2008 y 2010.

\section{Fuentes y datos}

Se tomó como fuentes los estados financieros de la Superintendencia de Sociedades y la Cámara de Comercio de Pereira de las empresas seleccionadas, que proyectaron sus estados financieros del 2008 y 2010. 
Tabla 1. Variable e indicadores utilizados para realizar el análisis de discriminante

\begin{tabular}{|ll|}
\hline Variable & Indicador \\
\hline RC & Razón corriente \\
PA & Prueba ácida \\
CN & Capital neto \\
MB & Margen bruto \\
MO & Margen operacional \\
MN & Margen neto \\
UO & Utilidad operacional \\
\hline
\end{tabular}

Fuente: Autores.

\section{Análisis}

Para el análisis de los datos de Tabla 1, se utilizó el Software SPSS Statistics 19. Seguidamente se procedió a verificar el cumplimiento de los supuestos requeridos para aplicar el Análisis Discriminante y de esta forma calcular y establecer la función objetivo.

\section{Resultado}

A continuación se muestran los resultados de la aplicación de análisis discriminante en las empresas certificadas de Pereira para la seguridad comercial y, su incidencia, en la liquidez y rentabilidad.

\subsection{Verificación de los supuestos}

En la Tabla 2 y Tabla 3, se presenta los resultados de Normalidad para los datos de los periodos 2008 y 2010, asociados a la prueba de Shapiro \& Wilk, que requiere que los estadísticos estén por encima de 0,8. Las pruebas de normalidad para las variables por separado de los dos periodos respectivos dan como resultado que algunas no se comportan como variables normales por lo que se viola el supuesto de multinormalidad.

El supuesto de igualdad de matrices de varianzacovarianza para los dos años 2008 y 2010 se comprobó con la prueba de Box, como se muestra en la Tabla 4.

Los resultados obtenidos del estadístico de contraste $M=170,972$ y un valor de $F=3,958$ con una probabilidad asociada $p=0,00$ lo cual permite rechazar con un alto grado de confianza la hipótesis nula de que no existen diferencias entre las matrices de covarianza de los dos grupos. Criterio que permite concluir que los indicadores estudiados en los dos periodos son significativamente diferentes.

\subsection{Selección de las variables que mejor discriminan}

Como se mencionó anteriormente el criterio de selección de las variables es el de la distancia $D^{2}$ de Mahalanobis, se utilizaron todas las variables seleccionadas, siendo las variables que mejor discriminan la Razón Corriente, la Prueba Ácida, el Margen Bruto, Margen Neto, y Margen Operacional en la evaluación del impacto de la

Tabla 2 y Tabla 3. Prueba de Shapiro \& Wilk para la comprobación de la normalidad de las razones financieras del año 2008 y 2010 respectivamente.

\begin{tabular}{|c|c|c|c|c|c|c|c|}
\hline \multicolumn{4}{|c|}{ Shapiro-Wilk 2008} & \multicolumn{4}{|c|}{ Shapiro-Wilk 2010} \\
\hline & Estadístico & GI & Sig. & & Estadístico & gl & Sig. \\
\hline $\mathrm{RC}$ & 0,706 & 12 & 0,001 & $\mathrm{RC}$ & 0,278 & 12 & 0,011 \\
\hline PA & 0,701 & 12 & 0,001 & $\mathrm{PA}$ & 0,275 & 12 & 0,013 \\
\hline $\mathrm{CN}$ & 0,505 & 12 & 0,000 & $\mathrm{CN}$ & 0,468 & 12 & 0,000 \\
\hline MB & 0,357 & 12 & 0,000 & $M B$ & 0,282 & 12 & 0,009 \\
\hline MO & 0,946 & 12 & 0,548 & MO & 0,417 & 12 & 0,000 \\
\hline MN & 0,548 & 12 & 0,000 & $\mathrm{MN}$ & 0,260 & 12 & 0,025 \\
\hline UO & 0,905 & 12 & 0,186 & UO & 0,353 & 12 & 0,000 \\
\hline
\end{tabular}


Tabla 4. Resultados de la prueba de BOX

\begin{tabular}{|c|c|c|}
\hline \multicolumn{2}{|c|}{ M de Box } & 170,972 \\
\hline \multirow{4}{*}{$F$} & Aprox. & 3,958 \\
\hline & gl1 & 28 \\
\hline & $g \mid 2$ & 1686,534 \\
\hline & Sig. & , 000 \\
\hline
\end{tabular}

certificación BASC en la liquidez y rentabilidad de las empresas de Medellín, constituyendo estas la funciones discriminante, el resultado final del modelo se muestra en la tabla 5 y en las ecuaciones (4) y (5).

Con base en los resultados anteriores se procedió a calcular la función discriminante de las empresas certificadas de Pereira, los cuales muestran los indicadores que discriminan y la variabilidad del modelo de las organizaciones.

Tabla 5. Coeficientes de la funciones de clasificación

\begin{tabular}{|ccc|}
\hline Año & $\mathbf{2 0 0 8}$ & $\mathbf{2 0 1 0}$ \\
\hline $\mathrm{RC}$ & 3,210 & 1,856 \\
$\mathrm{PA}$ & $-2,849$ & $-1,332$ \\
$\mathrm{CN}$ & $-2,220 \mathrm{E}-8$ & $-1,931 \mathrm{E}-8$ \\
MB & 0,000 & 0,000 \\
MO & $2,968 \mathrm{E}-5$ & $4,584 \mathrm{E}-5$ \\
MN & $8,433 \mathrm{E}-5$ & $3,581 \mathrm{E}-5$ \\
UO & $1,205 \mathrm{E}-7$ & $1,186 \mathrm{E}-7$ \\
(Constante) & $-3,264$ & $-2,372$ \\
\hline
\end{tabular}

$$
\begin{aligned}
Z_{2008}= & 3,210^{*}(\mathrm{RC})-2,849^{*}(\mathrm{PA})-2,220 \mathrm{E}-8^{*}(\mathrm{CN})+ \\
& 2,968 \mathrm{E}-5^{*}(\mathrm{MO})+8,433 \mathrm{E}-5^{*}(\mathrm{MN})+ \\
& 1,205 \mathrm{E}-7^{*}(\mathrm{UO})-3,264 \\
\mathrm{Z}_{2010}= & 1,856^{*}(\mathrm{RC})-1,332^{*}(\mathrm{PA})-1,931 \mathrm{E}-8^{*}(\mathrm{CN})+ \\
& 4,584 \mathrm{E}-5^{*}(\mathrm{MO})+3,581 \mathrm{E}-5^{*}(\mathrm{MN})+ \\
& 1,186 \mathrm{E}-7^{*}(\mathrm{UO})-2,372
\end{aligned}
$$

La capacidad de clasificación del modelo presenta para año 2008 58,3\% y para año $201041,7 \%$, para una efectividad de clasificación de $66,7 \%$, como se muestra en la Tabla 6.

\subsection{Evaluación de los Indicadores seleccionados en las empresas certificadas en BASC en Pereira}

Cuando se revisan los estadísticos (Media) de los indicadores seleccionados de las empresas en Pereira, podemos observar que existe un impacto de la certificación BASC en los indicadores de Razón Corriente (RC), Prueba Acida (PA), Margen Bruto (MB) y Margen Operacional (MO), lo que no ocurre para los indicadores de Capital Neto (CN), Margen Neto (MN) y Utilidad Operacional (UO), los cuales no presentan variación significativa que mejore las operaciones en materia financiera del sector, como se muestra en la Tabla 7.

\section{Discusión}

Al analizar las funciones discriminantes generadas en la Tabla 5, se puede analizar que indicadores como la Prueba Ácida (PA) y el Capital Neto (CN), discriminan bien mejorando de un periodo a otro. Los indicadores de Razón Corriente $(\mathrm{RC})$ y Margen Neto $(\mathrm{MN})$ discriminan forma negativa, es decir, disminuyen sus indicadores de un periodo a otro, mientras que los indicadores Margen Bru-

Tabla 6. Resultados de la clasificación

\begin{tabular}{|cccccc|}
\hline & Año & Grupo de pertenencia pronosticado & Total \\
\hline \multirow{3}{*}{ Original } & & 2008 & 2010 \\
& & & & 5 & 12 \\
& & 2008 & 7 & 9 & 12 \\
& Recuento & 2010 & 3 & 41,7 & 100,0 \\
& Porcentaje & 2008 & 58,3 & 75,0 & 100,0 \\
\hline
\end{tabular}


Tabla 7. Medias de los indicadores financieros

\begin{tabular}{|c|c|c|c|}
\hline Año & $\begin{array}{l}\text { Indicador } \\
\text { Financiero }\end{array}$ & Media & Desv. típ. \\
\hline \multirow[t]{7}{*}{2008} & $\mathrm{RC}$ & 2,7789 & 2,85030 \\
\hline & PA & 1,8739 & 1,98761 \\
\hline & $\mathrm{CN}$ & 27430616,9167 & 60583202,56941 \\
\hline & MB & 3077,2852 & 8684,41167 \\
\hline & MO & 690,6706 & 1145,96112 \\
\hline & $\mathrm{MN}$ & 5426,7508 & 14091,86117 \\
\hline & UO & 7607877,3333 & 10063701,63983 \\
\hline \multirow{7}{*}{2010} & $\mathrm{RC}$ & 2,9092 & 2,78591 \\
\hline & PA & 2,2454 & 2,78393 \\
\hline & $\mathrm{CN}$ & 25390445,7500 & 73578010,84538 \\
\hline & MB & 480,0477 & 337,37333 \\
\hline & MO & 10404,8533 & 30653,88268 \\
\hline & $\mathrm{MN}$ & $-302,4735$ & 12421,65727 \\
\hline & UO & 7616294,0833 & 14259616,38288 \\
\hline
\end{tabular}

to (MB), Margen Operacional (MO) y Utilidad Operacional (UO) no aportan ninguna discriminación entre los grupos estudiados. Esto último sustenta la hipótesis de la poca capacidad de predicción del modelo obtenido.

En lo que respecta a las medias presentadas en la Tabla 7 de los indicadores seleccionados, se puede analizar que los indicadores Razón Corriente (RC), Margen Bruto (MB) y Margen Operacional (MO) mejoraron de un periodo a otro, por lo que podemos concluir que la certificación BASC impacta significativamente en los resultados operacionales dada la relación de proporcionalidad que existe estos indicadores. De la relación de las variables utilidad bruta/ ingresos operacionales y utilidad operacional/ingresos operacionales, podemos analizar que la mejora se genera en un indicador que esta relacionado directamente con las variables asociadas a un estándar como el BASC, el cual se centra específicamente en procesos operacionales. Sin embargo, en los otros indicadores no se observa una incidencia de la certificación en el mejoramiento de estos indicadores en el periodo estudiado 2008-2010.

Es importante mencionar que en este artículo se presenta un método y un conjunto de herramientas para la evaluación de la evolución de los diferentes indicadores financieros en las empresas de Pereira que se certificaron en BASC. Además se presenta una explicación clara de cuáles indicadores seleccionados mejoran de un periodo a otro. Cabe señalar que estudios similares (Fontalvo,
Mendoza \& Morelos, 2011) han demostrado que el indicador Razón Corriente, es sensible y muestra mejora significativas como efecto de la gestión en el sector estudiado, afirmación que se validad en esta investigación, teniendo en cuenta la variación positiva de este indicador en el procesos de certificación objeto de análisis.

\section{Conclusión}

En el Análisis Discriminante desarrollado en esta investigación para la construcción del modelo se utilizaron 60 empresas de Pereira, el modelo presentó una buena efectividad en la clasificación. En la muestra original la precisión del modelo en el 2008 es 58,3\% y en el 2010 es del $41,7 \%$, para un promedio total de clasificación de $66,7 \%$ de las empresas. Lo que demuestra la poca confiabilidad para predecir el comportamiento de los indicadores financieros en el sector a futuro. Sin embargo para otros sectores, estándares o Indicadores podría tener otra incidencia.

De la funciones discriminantes obtenidas y de los estadísticos analizados se puede concluir que la certificación BASC, incide positivamente en los indicadores financieros Razón Corriente (RC), Margen Bruto (MB) y Margen Operacional (MO). A partir de estudios como este, se podrá realizar análisis en diferentes sectores empresariales que faciliten la toma de decisiones sobre que variables, rubros e indicadores que redunden en el mejoramiento de la situación financiera de las organizaciones.

Se recomienda para futuros estudios incrementar el número de indicadores financieros e incorporar indicadores de productividad y competitividad; se invita analizar el comportamiento de otros sectores empresariales, para de esta forma tomar decisiones asociadas a las operaciones de las organizaciones que mejoren los estados financieros de las empresas.

\section{Referencias}

Cárcaba, Ana. «Análisis financiero de las entidades locales mediante el uso de indicadores". Revista española de financiación y contabilidad, 32(118)(2003): 661-692.

Criado, F. y Calvo, A. «Factores clave y rendimiento en empresas españolas con sistemas de gestión de la calidad". Revista Europea de Dirección y Economía de la Empresa, 18(2)(2009):107-126. 
Fernández, Juan. «Liderazgo empresarial en tiempos de cambio según Sun Tsu». Harvard Deusto Business Revierw, 8(109)(2002):42-48.

Fernández, E.; Jurado, P; Mas, O.; Navío, A. y Ruiz, C. «Implicaciones de la evaluación de impacto: Una experiencia en un programa de formación de formadores". Bordón. Revista de Pedagogía, 60(1)(2008):163-186.

Fontalvo, T.; Mendoza, A. y Morelos, J. «Evaluación del impacto de los sistemas de gestión de la calidad en la liquidez y rentabilidad de las empresas de la Zona Industrial de Mamonal (Cartagena, Colombia)». Revista Virtual Universidad Católica del Norte, 1(34)(2011):314-341.

Lachenbruch, Peter. Discriminant Analysis. New York: Hafner Press, 1975.

Martínez, Mario. Ideas Para el Cambio y el Aprendizaje en la Organización, una Perspectiva Sistémica. Bogotá: Ecoe Ediciones, 2005.

Mateos, R.; Iturrioz, J. y Gimeneo, R. «La participación financiera y el papel de la mujer en la toma de decisiones de las sociedades cooperativas: los consejos de administración». Revista Europea de dirección y economía de la empresa, 18(3)(2009):65-82.

Mileris, Rièardas. «Estimation of loan applicants default probability applying discriminant analysis and simple Bayesian classifier". Economics and management $=$ Ekonomika ir vadyba, 15(2010):1078-1084.

Mylonakis, J. y Diacogiannis, G. «Evaluating the likelihood of using linear discriminant analysis as a commercial bank card owners credit scoring model». International Business Research, 3(2)(2010):9-20.

Ortiz, Héctor. Análisis financiero aplicado y principios de administración financiera. Colombia: Universidad Externado de Colombia, 2011:118 p.

Ovalle, Mauricio y Ospina, Diana. Caracterización de las Empresas de Manizales Certificadas con la Norma BASC. Revista Ingeniería Industrial. Actualidad y Nuevas Tendencias, 1(3)(2009):20-30.

Peretto, Claudia. «Utilización del análisis discriminante logístico para explorar las causas de la eficiencia del sistema bancario Argentino". Cuaderno de Cimbage, 11(4) (2009):39-57.
Pérez, Cesar. Técnicas de análisis de datos con SPSS 15. España: Pearson Prentice Hall, 2009:314 p.

Rodríguez, Jorge y Moreno, Álvaro. Fragilidad financiera de las firmas en Colombia, 2000 - 2006. Un análisis discriminante de un modelo Minskano. Documentos FCE, 2011: [consultado el 21 de noviembre de 2011]. Disponible en: <http:// bdigital.unal.edu.co/769/1/Doc_FCE_ EE_8.pdf > ISSN 2011-6322.

Sanchis, A.; Gil, J. y Heras, A. «El análisis discriminante en la previsión de la insolvencia en las empresas de seguros de no vida». Revista Española de financiación y contabilidad, 32(116)(2003):183-233.

Suárez, Javier. «Los parámetros característicos de las empresas manufactureras de alta rentabilidad. Una aplicación del análisis discriminante». Revista Española de financiación y contabilidad, 29(104)(2000):443-481.

Superintendencia de Sociedades: [consultado el 01 de octubre de 2010] Disponible en: <http://sirem. super sociedades.gov.co/SIREM/ >

Tabachnick, B. G. y Fidell, L. S. Using Multivariate Statistics. Boston: Allyn \& Bacon, 2007.

Tejada, J. y Fernández, L. «La evaluación del impacto de la formación como estrategia de mejora en las organizaciones». Revista Electrónica de Investigación Educativa, vol. 9, No. 2: [consultado el 26 de noviembre de 2011] Disponible en: <http://redie.uabc.mx/vol9no2/contenidotejada2.html >ISSN 1607-4041, 2007.

Vásquez, X.; Guerra, A. y Ahmed, I. Aplicación de métodos multivariados: una respuesta a las limitaciones de los ratios financieros: [consultado el 20 de octubre de 2011] Disponible en: < http://www.eumed.net/ce/2011a/ >, 2008.

Vuran, Bengü. «Prediction of business failure: A comparison of discriminant and logistic regression analyses". Istanbul University journal of the school of business administration, 38(1)(2009):47-65.

World BASC Organization, WBO. Beneficios, Quienes Somos: [consultado el 15 de septiembre de 2011] Disponible en: <http://www.wbasco.org/espanol/quienes somos.htm />. 2011. 